\title{
Proyectos de Aula Semestrales como Estrategia Pedagógica para la Formación en Ingeniería
}

\author{
José D. Torres, Diofanor Acevedo y Piedad M. Montero \\ Universidad de Cartagena, Facultad de Ingeniería, Programa de Ingeniería de Alimentos, Avenida el \\ Consulado, Calle 30 No. 48-152. Cartagena, Bolívar-Colombia (e-mail: jtorresg3@unicartagena.edu.co)
}

Recibido Abr. 7, 2015; Aceptado Jun. 24, 2015; Versión final Ago. 6, 2015, Publicado Jun. 2016

\begin{abstract}
Resumen
El objetivo de este trabajo fue analizar la utilización de proyectos de aula como estrategia para la formación profesional en Ingeniería. A un curso de 20 estudiantes, con subgrupos de dos o tres individuos se le asignó la realización de un proyecto de aula durante todo el semestre. El docente actuó como un orientador y la evaluación se realizó por medio de trabajos escritos y orales. Se aplicó un cuestionario para verificar la percepción de los estudiantes sobre ésta estrategia pedagógica utilizada. El análisis de los resultados mostró que la metodología utilizada fue importante para el aprendizaje de los estudiantes, ya que fomentó el autoaprendizaje, la creatividad y la solución de problemas reales. La implementación de proyectos de aulas es una alternativa viable para la formación de estudiantes de ingeniería.
\end{abstract}

Palabras clave: trabajo semestral; proyecto de aula; autoaprendizaje; ingeniería; pedagogía; aprendizaje

\section{Term Classroom Projects as Pedagogical Strategy for Engineering Education}

\begin{abstract}
The objective of this study was to analyze the use of term classroom projects as a strategy for vocational formation of engineering students. A course of 20 students, with subgroups of two or three persons was assigned the development of a classroom project during the whole semester. The instructor acted as an advisor and the evaluation was conducted through written and oral tests. A questionnaire was applied to verify the perception of the students on the new educational strategy. The analysis of the results showed that the methodology was important for student learning because it encouraged self-learning, creativity and solving real problems. The implementation of term classrooms projects is a viable option for further training of engineering students.
\end{abstract}




\section{INTRODUCCIÓN}

El proyecto de aula es una propuesta metodológica que permite reunir los objetivos de las unidades de aprendizaje en un semestre para la solución de un problema, estableciendo unos indicadores alcanzables por los estudiantes, y que a su vez incentivan el desarrollo de distintas destrezas del pensamiento, abriendo nuevas oportunidades para impulsar orientaciones innovadoras de enseñanza (Forero et al., 2002; QuirogaRamírez, 2012; Benítez y García, 2013). Los proyectos de aula se consideran como una estrategia de la planificación e instrucción válida para reorientar la práctica pedagógica en sus diferentes componentes, implicando un cambio de paradigma a partir de principios orientadores hacia una práctica constructiva e integral capaz de transformar al individuo (Rodríguez y Cortés, 2010; Morales y Torres, 2015).

Ballesteros y Moral (2014) y Valderrama y Valderrama (2014) señalan que para atender a varias necesidades se han implementado diferentes habilidades pedagógicas, destacándose entre ellas los proyectos de aula o también llamados trabajos semestrales guiados; los cuales buscan aprovechar los conocimientos recopilados en las asignaturas de Ingeniería, sobre un proceso o producto específico, a través del cual el estudiante va adquiriendo la capacidad de relacionar los conceptos teóricos con la experiencia práctica para solucionar problemas reales (Barrientos, 2012; Canales y Schmal, 2013). El tipo de aprendizaje a través de la implementación de proyectos se denomina "aprendizaje basado en proyectos" (Gonçalves, 2014). Con la aplicación de dicha estrategia los estudiantes definen el propósito, identifican su mercado, investigan la temática, crean un plan de gestión, diseñan y desarrollan un producto o proceso (Hartmann y Dorée, 2015).

En la educación fundamentada en propósitos, los docentes necesitan crear espacios para el aprendizaje, dando acceso a la información, soportando la enseñanza por la instrucción, modelamiento y guía a los estudiantes, para manejar de manera apropiada sus tareas, animarlos a utilizar procesos de aprendizaje meta-cognitivos, respetar los esfuerzos grupales e individuales, verificar el progreso, diagnosticar problemas, dar retroalimentación y evaluar los resultados generales (Rodríguez-Sandoval y CortésRodríguez, 2010; Martínez, 2015). Si bien es cierto, el objetivo principal de estos proyectos es dejar que los estudiantes se incluyan como seres activos, empleando un lenguaje real y desarrollando la motivación, la participación, el trabajo en grupo, el rendimiento académico, el fomento de la lectura y la escritura como las claves principales del aprendizaje (Oses y Carrasco, 2013). La felicidad en los estudiantes tiene potenciales resultados en el rendimiento, puesto que existe un efecto positivo, la relación entre la creatividad, la felicidad y el rendimiento académico, ha sido asociado positivamente con la permanencia de los estudiantes en los primeros años de estudio (Jabbaz y Samper, 2013; Ramírez y Fuentes, 2013; Acevedo et al., 2015). Esto apoya la idea que el desempeño académico del estudiante es el resultado tanto de sus conocimientos y habilidades (Ramírez y Fuentes, 2013). Por esta razón, los proyectos ocupan los principios más importantes de las comunidades, como el aprovechamiento de los conocimientos de cada persona, el seguimiento de revisar lo que se está haciendo para llegar a ello, respetando las contribuciones de los demás y aprendiendo de los errores (Gallardo-Camacho, 2013).

Por consiguiente los proyectos se han convertido en un camino competente para elevar los aprendizajes de los estudiantes, en todas las áreas del conocimiento, especialmente en Ingeniería, reconociendo su importancia como estrategia metodológica para el desarrollo de capacidades y destrezas necesarias en el mundo actual (Fernández y Duarte, 2013). El trabajo de los estudiantes en proyectos se considera una estrategia potente para la formación de un pensamiento globalizado, donde se combinan estructuras, procedimientos, formas de razonamiento, es decir representa la construcción personal (Benítez y García, 2011). Otra de las bondades que ofrecen los proyectos es que ayuda a aumentar la creatividad, en el sentido de la flexibilidad para enfrentar diversas tareas (Benítez y García, 2013). Evidentemente es importante destacar que uno de los beneficios que trae la utilización de proyectos en el aula de clase es el trabajo y aprendizaje colectivo (Delceva, 2014; Ballesteros y Moral, 2014). Archera et al., (2014) afirma que el alumno que realiza un proyecto de aula se instruye mejor que los otros compañeros de grupo que no lo realizan. De igual forma Quiroga-Ramírez (2012) señala que los proyectos promueven la forma de pensar, incrementando la creatividad e imaginación en los estudiantes ofreciéndoles experiencias significativas a su vida cotidiana; en otras palabras, con los proyectos los estudiantes utilizan su capacidad de crear e inventar, así favorecen los diferentes estilos de aprendizaje que cada uno posee.

El proceso de enseñanza debe concebirse entonces como progresivo y en constante movimiento, desarrollado por estrategias pedagógicas entendiendo la pedagogía como una mediación que promueve en los educandos la tarea de construir y apropiarse de sí mismos (Morales y Torres, 2015). El aprendizaje por proyectos en Ingeniería, resulta una estrategia imprescindible para lograr una enseñanza significativa y pertinente, constituyendo una estrategia educativa integral que contribuye en las fortalezas individuales de los estudiantes permitiendo explorar sus áreas de interés (González y Ramírez, 2011). La formación del Ingeniero hoy en día, es un desafío debido a la gran cantidad de información disponible y la complejidad 
creciente de los problemas; existe una preocupación por parte de las Universidades y docentes, por desarrollar alternativas y estrategias pedagógicas y didácticas que permitan la formación de profesionales en Ingeniería con las competencias exigidas por los entornos sociales actuales, como: el aprender a aprender, organizar y planificar, analizar y sintetizar, y trabajar de forma colaborativa con distintos profesionales (Fernández y Duarte, 2013; Acevedo et al., 2015). El objetivo de este trabajo fue analizar la utilización de un proyecto de aula como una estrategia para la formación integral de estudiantes de último semestre en un programa de Ingeniería.

\section{MATERIALES Y MÉTODOS}

\section{Estrategias pedagógicas}

Al comenzar el periodo académico, se le planteó a un curso de 20 estudiantes de último semestre en un programa de Ingeniería, la realización de un proyecto de aula, para desarrollarlo durante el semestre académico, y donde aplicaron los conceptos teóricos vistos en clases y asignaturas previas, como matemáticas, geometría, cálculo, física, diseño de equipos y operaciones unitarias, entre otras. Las opciones temáticas para los estudiantes fueron de libre escogencia, según sus propias inclinaciones. También se les brindó la oportunidad de que el profesor le sugiriera el tema del proyecto de aula de acuerdo a las competencias e intereses de cada alumno. Los grupos estuvieron conformados por 2 o 3 estudiantes, dependiendo del nivel de complejidad del tema escogido. Con el desarrollo de los proyectos de aula se buscó solucionar alguna problemática de tipo ecológico o social, así como fomentar la independencia, autoformación y autocrítica de los estudiantes, fundamentados en los lineamientos del aprendizaje basado en proyectos y la pedagogía crítica (Peñalonzo, 2011; Morales y Torres, 2015).

Después de seleccionar la temática, los estudiantes debieron justificar su respectivo estudio por medio de información económica, verificando el mercado, la viabilidad y la importancia técnica del proyecto, y su posible impacto a nivel social. A continuación, cada grupo de trabajo estableció sus objetivos, realizando y organizando la planeación experimental teniendo en cuenta los materiales y los métodos que iban a utilizar, así como las fuentes de información consultadas. Una vez reunieron toda la información coordinaron las respectivas actividades y asesorías con el docente, el cual actuó como un orientador a los equipos de trabajo, dando recomendaciones, formulando interrogantes, ayudando al grupo en el proceso de toma de decisiones y permitiendo que los estudiantes desarrollaran su tarea de forma independiente. Posteriormente el docente realizó pruebas preliminares para ajustar la metodología y los objetivos propuestos de cada grupo, y después ayudar a organizar y ejecutar la experimentación final del proyecto.

Del grupo de estudiantes y de los proyectos asignados se escogió uno de muestra para poder analizarlo en detalle. Debido a que se consideró como el de mayor complejidad, puesto que intentó solucionar una problemática de tipo ecológica, ya que implicó el diseño y la construcción de una máquina eléctrica piloto automatizada con registro de temperaturas, para realizar el proceso de cocción por ebullición de un producto alimenticio tradicional en la Región Caribe Colombiana, elaborado a partir de masa de maíz verde, conocido como "Bollo de Mazorca" que comúnmente se cocina de manera artesanal en recipientes metálicos y expuesto al aire libre en fogones de leña; es decir en condiciones que pueden llegar a generar componentes tóxicos al medio, porque implican la quema de madera para generar el fuego necesario para la cocción. A los estudiantes se les asignó un horario de atención con el docente tutor para comentar los respectivos avances y desarrollo experimental en el proyecto de aula escogido.

\section{Métodos de evaluación del proyecto de aula}

La evaluación se realizó por medio de trabajos escritos y orales de la siguiente manera: 1) La propuesta inicial con planteamiento del problema, y justificación (15\% en la nota final). 2) Realización de los objetivos y metodologías (15\%). 3) Entrega de avances escritos y realización de pruebas experimentales (20\%). 4) Presentación oral final (25\%). 5) Reporte del informe escrito final del proyecto (25\%). Estos dos últimos fueron los que tuvieron un mayor valor porcentual porque fue la síntesis general de toda la vivencia experimental del semestre. El formato del reporte final escrito siguió la presentación normal de un trabajo científico constituido por título, autores, resumen, introducción, objetivos, materiales y métodos, resultados y discusión, conclusiones y referencias, con tablas y figuras.

\section{Instrumento de la Investigación}

La evaluación de la estrategia pedagógica se llevó a cabo aplicando un cuestionario, similar a lo propuesto por Rodríguez-Sandoval y Cortes-Rodríguez (2010). Y donde se indagó sobre la percepción que tuvieron los estudiantes en la forma como se desarrolló y evalúo el proyecto. Esta encuesta fue realizada al finalizar el segundo semestre del 2014 con todos los estudiantes participantes del curso. Las preguntas de la encuesta fueron cerradas y se contestaron marcando "si" y "no". Además, se dejó un espacio al final para que estos expresaran sus comentarios generales y sugerencias sobre las estrategias utilizadas. 


\section{Análisis de los datos}

Los resultados se analizaron empleando Microsoft Excel® para Windows 2013, y posteriormente el Programa Estadístico para Ciencias Sociales, IBM SPSS (c) - versión 19.0. Para realizar los análisis con un nivel de confianza del $95 \%$.

\section{RESULTADOS Y DISCUSIÓN}

A continuación se detalla parte los resultados del proyecto de mayor complejidad entre los realizados por los estudiantes. El cual llevó por título: "Diseño y construcción de una maquina eléctrica automatizada con control de temperaturas para la cocción por ebullición de un producto a base de maíz "Bollo de Mazorca".

Parte 1. Proyecto diseño y construcción de la maquina eléctrica automatizada para la cocción por ebullición

Para cálculo del volumen de la máquina se determinó primero el tamaño del recipiente partiendo del diámetro, altura y la forma. De acuerdo al producto se tomó una relación $1: 1$ entre el diámetro y la altura. El recipiente tuvo una forma cilíndrica. Dónde: $\mathrm{D}=$ Diámetro $=25 \mathrm{~cm}$ y h=Altura $=25 \mathrm{~cm}$. Como se muestra en la Figura 1.

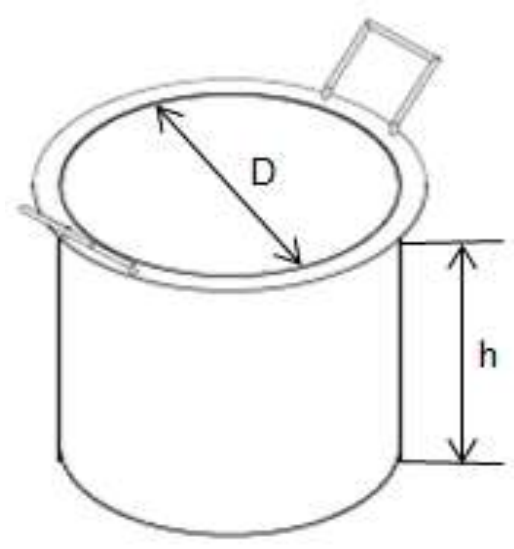

Fig. 1: Esquema del recipiente de la máquina eléctrica automatizada para la cocción por ebullición

El volumen del recipiente aproximadamente fue 12,3 litros, y el mínimo requerido para hacer la cocción por ebullición fue de 5,0 litros. De tal manera que el operador pudiera realizar pruebas cómodamente. La resistencia eléctrica seleccionada fue de $1,10 \mathrm{~W}$ con un voltaje nominal a $110 \mathrm{~V}$, debido a su compatibilidad con la red doméstica además de ser muy comercial y asequible en cuanto al precio. Además aseguró un rápido calentamiento del agua sin grandes pérdidas de calor. El riesgo de accidentes por sobrecalentamiento fue mínimo con respecto a una resistencia de mayor potencia.

Para el cálculo de la transferencia de calor hacia el metal y hacia el agua, se realizó un balance energético, como se indica en la ecuación 1.

$\Delta Q=(M m)\left(C_{P} a c\right)\left(\Delta^{\circ} T\right)+\left(M_{A}\right)\left(C_{p}\right)\left(\Delta^{\circ} T\right)$

Dónde: $\Delta \mathrm{Q}=$ calor suministrada por la resistencia. $\mathrm{M}_{\mathrm{m}}=$ masa de la lámina de acero; $\mathrm{C}_{\mathrm{p} . \mathrm{ac}}=$ calor específico del acero a $500 \mathrm{~J} / \mathrm{Kg}{ }^{\circ} \mathrm{C} ; \Delta^{\circ} \mathrm{T}=$ diferencia de temperaturas $95^{\circ} \mathrm{C}-28^{\circ} \mathrm{C}=67^{\circ} \mathrm{C}$. MA = masa del agua; $\mathrm{Cp}$ $=$ calor específico del agua. La resistencia transfiere 1,10W. Por lo tanto:

$\Delta Q=(1 \mathrm{Kg})\left(500 \frac{\mathrm{J}}{(\mathrm{Kg})\left({ }^{\circ} \mathrm{C}\right)} \cdot \mathrm{Acer}\right)\left(67^{\circ} \mathrm{C}\right)+(5000 \mathrm{~g})\left(4,186 \frac{\mathrm{J}}{(\mathrm{Kg})\left({ }^{\circ} \mathrm{C}\right)}\right)\left(67^{\circ} \mathrm{C}\right)=1,436 \mathrm{KJ}=1436,1 \mathrm{~J}$

Luego:

$\Delta t=\left(\frac{1436,1 \mathrm{~J}}{1,10 \mathrm{~W}}\right)=1305,54 \mathrm{~s}=21,75$ minutos

Los 5 litros de agua alcanzaron los $95^{\circ} \mathrm{C}$ en un tiempo aproximadamente de 21,75 minutos. De acuerdo a las normas de fabricación de recipientes para alimentos, así como recomendaciones de fabricantes con mucha experiencia y teniendo en cuenta los costos, se optó por seleccionar el acero inoxidable 304 como mejor solución para la construcción del equipo. Ya que poseía las siguientes características: inoxidable, 
buena resistencia a la corrosión en el rango de hasta $300^{\circ} \mathrm{C}$ y $350^{\circ} \mathrm{C}$. No requiere un tratamiento posterior al proceso de soldadura; tiene propiedades para embutido profundo, no es templable ni magnético, puede ser fácilmente trabajado en frío, doblado, cilindrado además tiene buenas propiedades mecánicas. En la Figura 2 se muestra el diseño planteado durante la segunda fase, de la máquina de cocción automatizada con control de temperatura, la cual tuvo la mejor calificación.
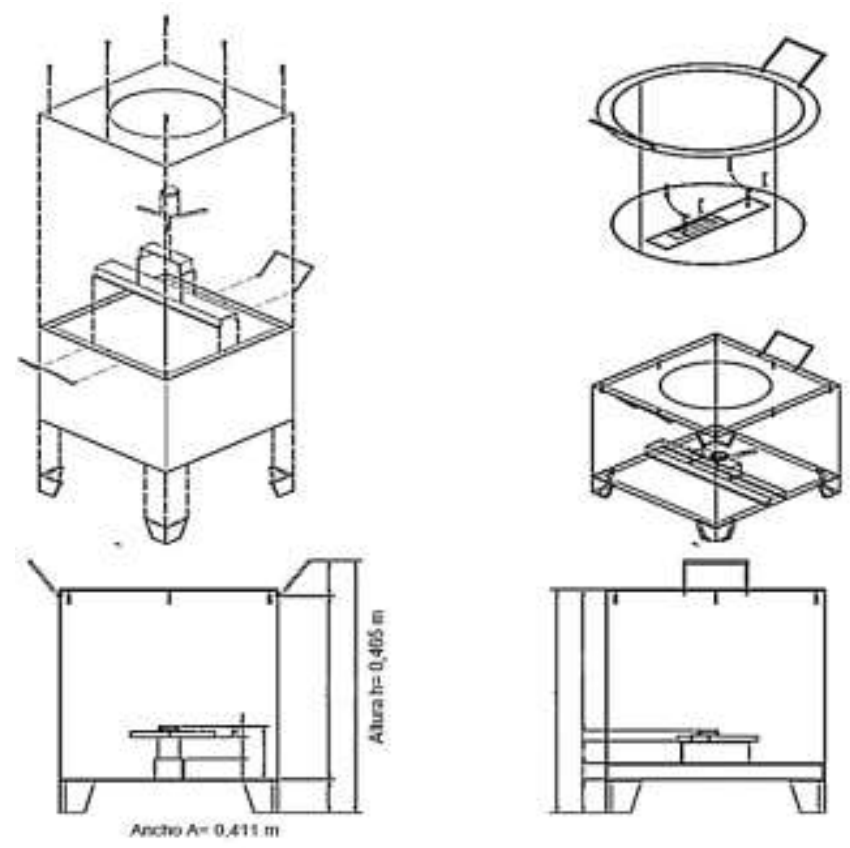

Fig. 2: Esquema de la maquina automatizada para la cocción por ebullición, con sus dimensiones

En la Figura 3, se esquematiza la maquina automatizada construida y acoplada con un sistema de adquisición de datos en un computador personal que registró las variaciones de temperatura de la muestra, e indicó el tiempo y la temperatura adecuada para procesar la muestra. Los componentes del prototipo son: a. Recipiente de cocción; b. Soporte de contención; c. Tablero de caja y con circuitos micro-controlados; d. Sensores.

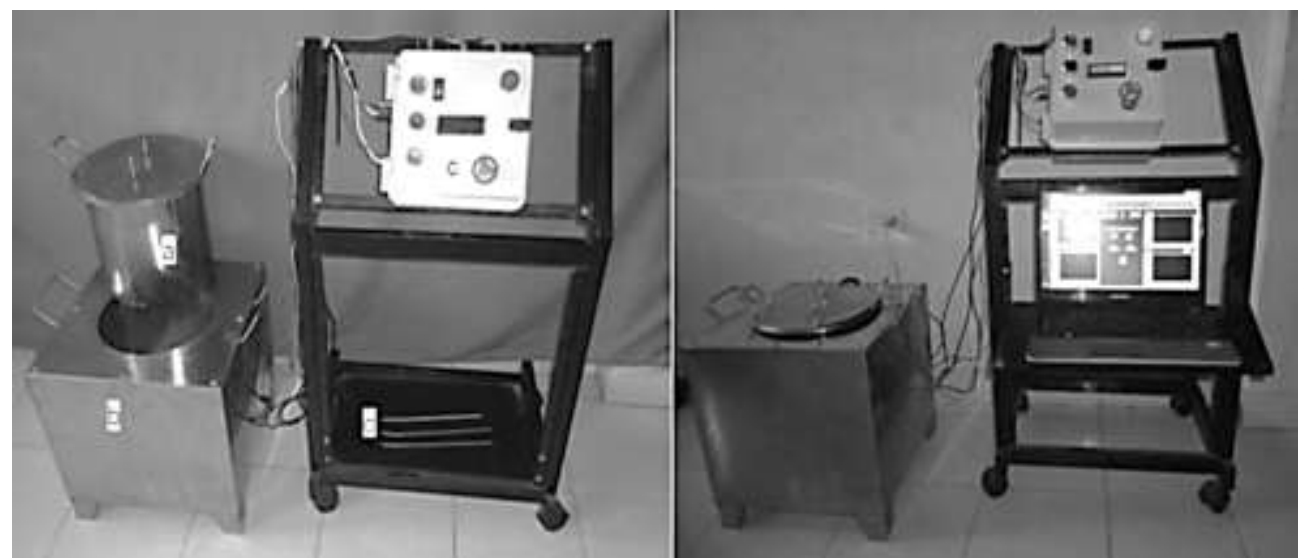

Fig. 3. Máquina de cocción por ebullición automatizada construida en la ejecución del proyecto de aula.

\section{Síntesis general del modo de operación de la maquina eléctrica construida}

El sistema es energizado con el fluido eléctrico de la red comercial, teniendo en cuenta que trabaje con un voltaje de $110 \mathrm{~V}$ a $60 \mathrm{~Hz}$. El operador debe accionar el interruptor de encender del equipo. Una vez encendido el sistema debe verificar el nivel de agua y que los sensores estén bien ubicados en el producto a procesar en este caso "Bollo de Mazorca". Para iniciar todo el proceso el operador debe accionar el interruptor que está en la caja de control para que se inicie la cocción. La limpieza del equipo se debe realizar de forma manual, de la siguiente forma: a) se desconecta el equipo de la fuente de alimentación. b) se saca el recipiente del soporte y se desecha el agua. c) se retiran los sensores del producto y se procede a sacar. d) se prepara una solución de agua y jabón, y se precede a lavar el recipiente. Teniendo en cuenta las especificaciones del fabricante del producto, para una adecuada desinfección. e) se enjuaga el recipiente con abundante agua fría. f) por último se introduce el recipiente dentro del soporte. 


\section{Parte 2. Datos de la encuesta aplicada a los estudiantes de Ingeniería}

En esta parte se analiza el nivel de percepción de los estudiantes sobre la metodología de los proyectos de aula en el curso de último semestre. El resultado de la encuesta se recopiló en la Tabla 1. En la primera pregunta se puede observar que el $81,44 \%$ de los estudiantes consideraron que la estrategia pedagógica del proyecto de aula fue un apoyo importante en su aprendizaje y desarrollo profesional.

Tabla 1. Resultados del cuestionario aplicado a los estudiantes de Ingeniería del último semestre

\begin{tabular}{|l|c|c|}
\hline \multirow{2}{*}{ Preguntas } & \multicolumn{2}{|c|}{ Respuestas ( $\mathrm{n}=20)$} \\
\cline { 2 - 3 } & Si (\%) & No (\%) \\
\hline 1. ¿Cree usted qué el proyecto de aula es importante para su formación profesional? & 81,44 & 18,56 \\
\hline 2. ¿Fue difícil encontrar una idea de proyecto apropiado para desarrollar en el curso? & 53,98 & 46,02 \\
\hline 3. ¿Aplicaron los conceptos vistos en clase en la solución del proyecto de aula? & 72,33 & 27,67 \\
\hline 4. ¿Cree usted que la forma de realizar el proyecto durante el semestre fue la adecuada? & 67,45 & 32,55 \\
\hline $\begin{array}{l}\text { 5. ¿Está conforme con la forma de realizar los avances (entregas) del proyecto de aula durante } \\
\text { el semestre? }\end{array}$ & 48,16 & 51,84 \\
\hline 6. ¿Considera justo que la valoración final del proyecto sea la calificación final de la asignatura? & 54,77 & 45,23 \\
\hline 7. ¿El tiempo estipulado para el desarrollo experimental del proyecto es suficiente? & 33,84 & 66,16 \\
\hline $\begin{array}{l}\text { 8. ¿Este tipo de metodologías para el aprendizaje satisfacen sus expectativas como estudiante } \\
\text { comparándola con otros métodos tradicionales? }\end{array}$ & 57,29 & 42,71 \\
\hline $\begin{array}{l}\text { 9. ¿Considera que los materiales, equipos de laboratorios y demás recursos disponibles } \\
\text { actualmente, son los adecuados para el desarrollo de la parte experimental del proyecto? }\end{array}$ & 19,98 & 80,02 \\
\hline $\begin{array}{l}\text { 10. ¿Usted considera que la orientación para el desarrollo del trabajo por parte del docente } \\
\text { cumplió con sus expectativas? }\end{array}$ & 77,86 & 22,14 \\
\hline
\end{tabular}

Los resultados de esta investigación fueron similares a los reportados por Rodríguez-Sandoval y CortesRodríguez (2010) quienes señalan que es indispensable que en los proyectos que están orientados hacia el aprendizaje en Ingeniería, los estudiantes se motiven en el desarrollo y ejecución de los mismos, para que de esta manera puedan adquirir un aprendizaje valioso y efectivo. Así mismo con los hallados por Benítez y García (2013) quienes afirman que en Ingeniería es importante que los estudiantes desarrollen proyectos para estimular el autoaprendizaje, lo cual es indispensable debido a que estos son profesionales que estarán involucrados en el sistema productivo e industrial de la sociedad (Canales y Schmal, 2013).

En la segunda pregunta, el 46,02\% de los encuestados considero que fue difícil encontrar una idea de proyecto para desarrollar en el curso. Este resultado coincide con lo reportado por Morales y Torres (2015) quienes indican que es el reflejo de la falta de autonomía de muchos estudiantes para desarrollar un proyecto de acuerdo a sus inclinaciones académicas o profesionales, esta situación crea un poco de confusión en los estudiantes. Diversos autores como Oses y Carrasco (2013) señalan que el sistema educativo tradicional en ingeniería ha condicionado permanentemente a los estudiantes en el desarrollo de problemas y ejercicios de la literatura, sin prepararlos para problemas o situaciones reales, lo cual tiene más sentido si se considera que en un futuro van a formar parte de un sector productivo con repercusiones directas sobre la sociedad.

Quiroga-Ramírez (2012) afirma que los estudiantes que entran en la metodología de los proyectos de aula por lo general adquieren mayor autonomía y tienden a tener experiencias más positivas y a percibir mejor este tipo de aprendizaje. Lo que a su vez coincide con Gonçalves (2014) quien a su vez indicó que es importante que los profesores estimulen el aprendizaje de los alumnos al comienzo de la asignación de los proyectos de aula. Otros autores como Fernández y Duarte (2013) reportan que si los estudiantes están comprometidos y asumen correctamente este tipo de enseñanzas tendrán una importante experiencia profesional y desarrollo de habilidades meta-cognitivas. En la pregunta 3 se indagó por la aplicación de los conocimientos vistos en clase para la solución de los problemas del proyecto de aula, y solo el $27,32 \%$ de los encuestados respondieron negativamente. Por su parte Gallardo-Camacho (2013) investigó un proyecto de aula y comentó que estos son importantes ya que sitúan a los estudiantes en ambientes reales y contextualizados, y puede servir para construir puentes entre los conceptos teóricos de las temáticas del curso y las experiencias de la vida real. La forma como se desarrolló el proyecto de aula durante el semestre, se examinó en la pregunta 4. La mayoría de los estudiantes $(67,45 \%)$ consideraron que el proyecto de aula se aplicó en forma apropiada durante el curso. Esto último puede explicarse en base a lo que reportan Rodríguez y Cortés (2010) por el número de entregas o avances del proyecto que se realizaron durante el semestre, pues varios manifestaron la necesidad de incrementar este tipo de trabajos para afianzar y mejorar el desarrollo experimental del proyecto. 
La pregunta 5 se relaciona con la apreciación sobre la realización de los avances (entregas) del proyecto durante el semestre. El $51,84 \%$ de los encuestados aprobó este tipo de metodología. Los informes que presentaron los estudiantes a medida que avanzaban en el proyecto demostraron como mejoraron la capacidad para sintetizar y analizar los resultados que obtenían parcialmente. En la pregunta 6 se investigó la percepción que tuvieron los estudiantes sobre la valoración final del proyecto como calificación final en la asignatura, en lo cual un $54,77 \%$ estuvo en acuerdo con esta metodología, mientras que el $45,23 \%$ mencionó que es necesario complementarla con mayor número de talleres, exposiciones.

Delceva (2014) señala que la aplicación de los proyectos de aula es una herramienta para mejorar la comunicación entre los estudiantes y los docentes de Ingeniería. Sin embargo, los profesores deben tener cuidado en la utilización de estas técnicas para diagnosticar la comprensión del estudiante debido a que son menos estructuradas, más subjetivas y conllevan más tiempo. La evaluación del tiempo para el desarrollo experimental del proyecto se determinó en la pregunta 7. La mayoría de los estudiantes $(66,16 \%)$ consideraron que el tiempo para el desarrollo de la parte práctica del proyecto no fue suficiente debido principalmente a los problemas logísticos, la compra de los materiales y tiempo de dedicación académica. Los inconvenientes que se presentaron durante el desarrollo de los proyectos se utilizaron como herramientas para adquirir el conocimiento y potenciar las habilidades de los futuros profesionales en Ingeniería. Lo esencial que se buscó fue que los estudiantes aprendieran a analizar e interpretar datos experimentales con el fin de solucionar problemas representativos, siendo este el punto central del proyecto de aula. Benítez y García (2011) indican que los proyectos de aula potencian en los estudiantes principalmente las habilidades de investigación, así como la destreza de elaborar conclusiones.

La comparación entre la metodología de enseñanza tradicional y el aprendizaje obtenido mediante el proyecto de aula se llevó a cabo en la pregunta 8 . El 57,29\% de los estudiantes consideraron que los proyectos de aula como estrategia pedagógica satisfacían sus expectativas de aprendizaje en la asignatura. Lo cual fue similar a lo reportado por Ballesteros y Moral (2014) quienes señalan que los proyectos de aula deben ser un reto para los estudiantes, pero sin ser muy complejos. Los problemas requieren un balance de habilidades y desafíos que puedan generar una experiencia agradable en el aprendizaje. Los estudiantes que trabajan los proyectos de aula en pequeños grupos logran un mayor rendimiento académico, que cuando se emplean otros métodos de enseñanza (Valderrama, y Valderrama, 2014). Adicionalmente, adquieren mayores habilidades para el razonamiento y el pensamiento crítico, comprensión más profunda de la materia, menores niveles de estrés y ansiedad, mayor motivación y confianza para ver situaciones desde otras perspectivas, relación más positiva y de apoyo mutuo con compañeros, una mejor actitud hacia la materia y mayor autoestima (Hartmann y Dorée, 2015).

En la pregunta 9, se evaluaron los recursos disponibles en la Institución de educación superior para realizar la parte experimental del proyecto. El $80,02 \%$ de los estudiantes consideró que las condiciones de los equipos de los laboratorios no fueron los más apropiados para desarrollar la parte experimental del proyecto, ya que durante el semestre se presentaron inconvenientes logísticos, pero esto se utilizó como herramientas para mejorar la habilidad y la capacidad en la solución de problemas. Lo cual fue semejante a lo argumentado por Jabbaz y Samper (2013) quienes indicaron que los estudiantes deben desarrollar los proyectos de aula bajo condiciones adecuadas de instalaciones y de acceso de información, para de esta manera estimular la creatividad, el ingenio y la imaginación. En la pregunta 10 se evaluó el trabajo realizado por el profesor durante la estrategia pedagógica del proyecto de aula. Todos los estudiantes aprobaron la gestión realizada por el docente en el desarrollo de los proyectos planteados en el curso $(77,86 \%)$. En general Morales y Torres (2015) señalan que las nuevas aproximaciones cognitivas que representan los proyectos de aula requieren de un cambio sustancial del pensamiento y disposición de los profesores hacia las actividades, tareas y la estructura de la clase.

\section{CONCLUSIONES}

El proyecto de la maquina automatizada para la cocción por ebullición, permitió evidenciar el aprendizaje efectivo de los estudiantes del curso analizado, en cuanto a la síntesis y recopilación de los conocimientos aprendidos en las aulas de clases. Este proyecto se consideró el más completo puesto que implicó diseño, automatización, simulación, modelamiento, construcción y experimentación, actividades donde se requieren al máximo habilidades de pensamiento complejo y meta-cognitivas, indispensables para el desarrollo profesional de los Ingenieros. Los estudiantes estuvieron de acuerdo con la metodología puesto que consideraron que fomentó el autoaprendizaje, la creatividad, y el desarrollo de habilidades para solucionar problemas reales. Los estudiantes resaltaron la importancia de este tipo de estrategias para su formación profesional complementaria y consideraron que cumplió con sus expectativas de educación. La implementación de proyectos de aulas es una alternativa viable para la formación complementaria de estudiantes de ingeniería. 


\section{REFERENCIAS}

Acevedo, D., Torres, J., Tirado, D. "Análisis de la deserción estudiantil en el programa Ingeniería de Alimentos de la Universidad de Cartagena durante el periodo académico 2009 - 2013. Revista de Educación en Ingeniería, Formación Universitaria, 8(1) 35-42 (2015)

Archera, K. y otros seis autores, Examining the effectiveness of technology use in classrooms: A tertiary meta-analysis, Computers \& Education: 78(3) 140-149 (2014)

Ballesteros M. y A. Moral, Using simulation software to implement an active learning methodology in the university teaching, International Journal of Innovation in Education: 1 (1) 87-98 (2014)

Barrientos, M. y J.L. Navarrete, Aula de videojuegos un proyecto académico, Estudios sobre el mensaje periodístico: 18(1) 111-119 (2012)

Benítez A.A, y García M.L. Un Primer Acercamiento al Docente frente a una Metodología Basada en Proyectos, Formación Universitaria: 6(1) 21-28 (2013).

Benítez A.A, y M.L. García, Trabajos en Proyectos como Herramienta para Fortalecer las Habilidades Cognitivas en los Estudiantes, Formación Universitaria: 4(2) 27-36 (2011)

Canales, T. y R. Schmal, Trabajando con Pósteres: una Herramienta para el Desarrollo de Habilidades de Comunicación en la Educación de Pregrado, Formación Universitaria: 6(1), 41-52 (2013)

Delceva, J. Classroom management, International Journal of Cognitive Research in Science, Engineering and Education: 2(1) 51-55 (2014)

Fernández F.H. y J.E. Duarte, El Aprendizaje basado en Problemas como Estrategia para el Desarrollo de Competencias Específicas en Estudiantes de Ingeniería, Formación Universitaria: 6(5) 29-38 (2013)

Forero, E., A. Guerrero, G. López y M.C. Réquiz, El proyecto pedagógico de aula: Una utopía, una posibilidad o una realidad. Educere, Investigación: 5(16) 397- 404 (2002)

Gallardo-Camacho, J. Análisis de la integración del iPad en el aula desde la perspectiva del alumno: proyecto piloto de la UCJC, Historia y comunicación social: 18(1), 399-410 (2013)

Gonçalves, S.R, Preparing graduates for professional practice: findings from a case study of Project-based Learning (PBL), Procedia - Social and Behavioral Sciences: 139(1) 219 - 226 (2014)

González, M. y I. Ramírez, La formación de competencias profesionales: un reto en los proyectos curriculares universitarios, Revista Electrónica de Pedagogía: 8(16), 1-12 (2011)

Hartmann, A. y A. Dorée, Learning between projects: More than sending messages in bottles, International Journal of Project Management: 33 (2) 341-351 (2015)

Jabbaz, M. I. y M. T. Samper, El aula universitaria como empresa de investigación cualitativa, Revista d'innovació educativa: 11(1), 1 - 7 (2013)

Martínez, J., G. López., V. Rodríguez, y A. Martínez, El Aprendizaje Distribuido, Primero Paso a la virtualidad en la Enseñanza en el Nivel Universitario, Revista Iberoamericana de Producción Académica y Gestión Educativa: 2(36) 1-14 (2015)

Morales, C. y A. Torres, Aprendizaje Basado en Proyectos para el Desarrollo de Competencias, Revista Iberoamericana de Producción Académica y Gestión Educativa: 2(35) 1-10 (2015)

Oses, S.E y L. E. Carrasco, Módulos Alternativos en la Enseñanza de las Ciencias. Estrategia Didáctica Orientada al Logro de Aprendizajes Significativos, Formación Universitaria: 6(3), 39-52 (2013)

Quiroga-Ramírez, J. Potenciación del aprendizaje por medio del proyecto de aula con saberes transversales, Infancias Imágenes: 11 (1) 18-26 (2012)

Ramírez, P.E y C.A. Fuentes, Felicidad y Rendimiento Académico: Efecto Moderador de la Felicidad sobre Indicadores de Selección y Rendimiento Académico de Alumnos de Ingeniería Comercial, Formación Universitaria: 6(3) 21-30 (2013)

Peñalonzo, J. O. Pedagogía crítica y educación superior. Revista Educación, 26(2), 185-196 (2011)

Rodríguez-Sandoval, E. y M. Cortés-Rodríguez, Evaluación de la estrategia pedagógica "aprendizaje basado en proyectos". Percepción de los Estudiantes, Educación y Educadores, Revista Avaliação, Campinas, Sorocaba: 15(1) 143-158 (2010)

Valderrama, A. y J. Valderrama, Trabajo Semestral Guiado: un Buen Complemento al Estudio y Aprendizaje en Cursos de Ciencia y de Ingeniería, Formación Universitaria: 7(3) 3-10 (2014) 